\title{
Fluctuation-driven Coulomb drag in interacting quantum dot systems
}

\author{
Sierra, Miguel A.; Sánchez, David; Jauho, Antti-Pekka; Kaasbjerg, Kristen
}

Published in:

Physical Review B (Condensed Matter and Materials Physics)

Link to article, DOI:

10.1103/PhysRevB.100.081404

Publication date:

2019

Document Version

Publisher's PDF, also known as Version of record

Link back to DTU Orbit

Citation $(A P A)$ :

Sierra, M. A., Sánchez, D., Jauho, A-P., \& Kaasbjerg, K. (2019). Fluctuation-driven Coulomb drag in interacting quantum dot systems. Physical Review B (Condensed Matter and Materials Physics), 100, [081404(R)]. https://doi.org/10.1103/PhysRevB.100.081404

\section{General rights}

Copyright and moral rights for the publications made accessible in the public portal are retained by the authors and/or other copyright owners and it is a condition of accessing publications that users recognise and abide by the legal requirements associated with these rights.

- Users may download and print one copy of any publication from the public portal for the purpose of private study or research.

- You may not further distribute the material or use it for any profit-making activity or commercial gain

- You may freely distribute the URL identifying the publication in the public portal 


\title{
Fluctuation-driven Coulomb drag in interacting quantum dot systems
}

\author{
Miguel A. Sierra, ${ }^{1, *}$ David Sánchez, ${ }^{1}$ Antti-Pekka Jauho, ${ }^{2}$ and Kristen Kaasbjerg ${ }^{2, \dagger}$ \\ ${ }^{1}$ Instituto de Física Interdisciplinar y Sistemas Complejos IFISC (UIB-CSIC), E-07122 Palma de Mallorca, Spain \\ ${ }^{2}$ Center for Nanostructured Graphene (CNG), Department of Physics, Technical University of Denmark, DK-2800 Kgs. Lyngby, Denmark
}

(Received 6 March 2019; revised manuscript received 14 May 2019; published 13 August 2019)

\begin{abstract}
Coulomb drag between nanoscale conductors is of both fundamental and practical interest. Here, we theoretically study drag in a double quantum dot (QD) system consisting of a biased drive QD and an unbiased drag QD coupled via a direct interdot Coulomb interaction. We demonstrate that the Coulomb drag is driven by charge fluctuations in the drive QD, and show how the properties of the associated quantum noise allow one to distinguish it from, e.g., shot-noise-driven drag in circuits of weakly interacting quantum conductors. In the strong-interaction regime exhibiting an orbital ("pseudospin") Kondo effect, the drag is governed by charge fluctuations induced by pseudospin-flip cotunneling processes. The quenching of pseudospin-flip processes by Kondo correlations are found to suppress the drag at low bias and introduce a zero-bias anomaly in the second-order differential transconductance. Finally, we show that the drag is maximized for values of the interdot interaction matching the lead couplings. Our findings are relevant for the understanding of drag in QD systems and provide experimentally testable predictions in different transport regimes.
\end{abstract}

DOI: 10.1103/PhysRevB.100.081404

Introduction. In recent years, systems of closely spaced quantum dots (QDs) or nanoscale conductors have been demonstrated to be hosts of novel transport mechanisms which can be exploited in, e.g., quantum information [1], thermoelectrics [2-4], and energy harvesting [5]. Phenomena of fundamental importance such as, e.g., orbital Kondo physics [6-8] and attractive electron-electron interactions [9] have been demonstrated. For such effects, the Coulomb interaction between the constituents is essential, and its appreciable size in nanoscale systems has driven experiments into hitherto inaccessible regimes.

This development has led to a revival of the phenomenon of Coulomb drag [10] in nanoscale systems with several reports of drag currents-i.e., a current induced in an unbiased drag system by its Coulomb interaction with a biased current-carrying drive system-in Coulomb-coupled double quantum dot (DQD) systems in 2DEGs [11,12] as well as in graphene and carbon nanotubes $[13,14]$. Theoretically, drag in quantum conductors [15-18] and QD systems [12, 19-21] has been studied thoroughly, with indications of an intimate link $[17,22]$ between drag and the quantum noise of nonequilibrium fluctuations [23,24].

So far, theoretical works on drag in QD systems have been based on master-equation approaches in the Coulomb-blockade regime $\Gamma<k_{B} T, U$ [12,19-21], which do not apply to quantum coherent transport and provide no direct interpretation in terms of quantum noise. In addition, experiments have demonstrated Coulomb drag across different transport regimes [12,13], why further theoretical investigations may advance our understanding of

\footnotetext{
*msierra@ifisc.uib-csic.es

${ }^{\dagger} \mathrm{kkaa} @ \mathrm{dtu} . \mathrm{dk}$
}

Coulomb drag [12,13] and related transport effects [2,3,5], as well as the link to quantum noise.

In this Rapid Communication, we apply the Keldysh nonequilibrium Green's function (NEGF) formalism [25] to the description of Coulomb drag in QD systems across interaction regimes, covering weak $(\Gamma>U)$, to intermediate $(\Gamma \sim U)$, and strong $(\Gamma<U)$ Coulomb interactions $U$, where $\Gamma$ is the overall lead coupling.

As we here demonstrate, the leading contribution to the drag is governed by the nonsymmetrized quantum noise spectrum [23] of the nonequilibrium charge fluctuations in the
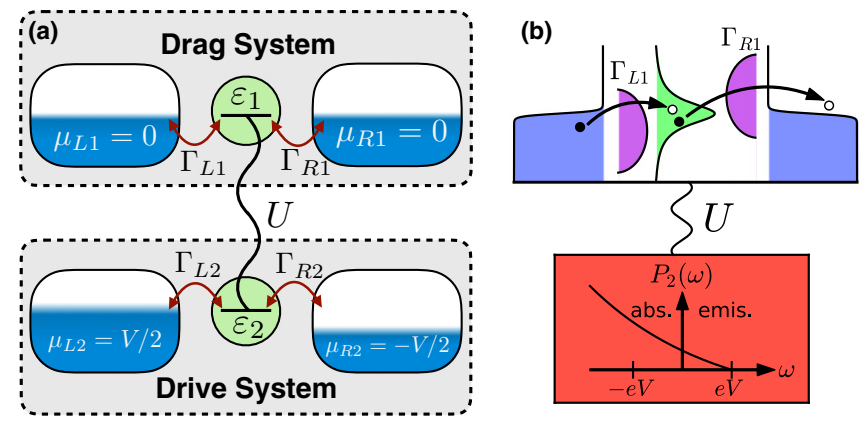

FIG. 1. (a) Schematic illustration of the double quantum dot setup consisting of an unbiased drag QD $(j=1)$ and a biased drive QD $(j=2)$ interacting via an interdot Coulomb interaction $U$ (no tunneling between the dots is allowed). (b) Energy diagram illustrating the charge-fluctuation-driven drag in Eq. (4). The quantum noise $P_{2}(\omega)$ of the charge fluctuations (with distinct absorption and emission components) in the biased drive system induces inelastic transitions in the drag system via the Coulomb interaction. This produces a drag current if the lead couplings $\Gamma_{L 1}$ and $\Gamma_{R 1}$ in the drag system have different energy dependencies. 
biased drive system,

$$
P_{2}(\omega)=\int d t e^{i \omega t}\left\langle\bar{n}_{2}(0) \bar{n}_{2}(t)\right\rangle,
$$

where $\bar{n}_{2}=\hat{n}_{2}-\left\langle\hat{n}_{2}\right\rangle$ is the occupation of the drive dot relative to its mean value (see also Fig. 1). This is in stark contrast to the drag induced between two coherent conductors [e.g., quantum point contacts (QPCs)] by a circuit environment [17], which is driven by the quantum shot noise in the drive system, i.e., current fluctuations whose noise characteristics are distinctly different from those of charge fluctuations [26]. This points to a fundamental difference between drag mediated by, respectively, direct Coulomb interactions (this study) and a circuit environment (Ref. [17]).

For strongly interacting DQD systems, higher-order processes involving tunneling events in both QDs become important, leading to an orbital analog [6-8] of the conventional spin Kondo effect [27-30] where the QD levels play the role of a pseudospin. To lowest order in the effective exchange coupling we find that the drag is dominated by pseudospin-flip cotunneling processes corresponding to simultaneous charge fluctuations in both QD systems. The quenching of charge fluctuations in the Kondo regime is found to suppress the drag at low-bias voltages, and leads to a zero-bias anomaly in the second-order derivative of the drag current with respect to the drive voltage.

Model and theory. We consider a spinless DQD system consisting of a drag $(j=1)$ and drive $(j=2)$ dot coupled via an interdot Coulomb interaction $U$ and connected to separate sets of source and drain contacts as depicted in Fig. 1. The total Hamiltonian takes the form $\mathcal{H}=\mathcal{H}_{\text {leads }}+\mathcal{H}_{\mathrm{DQD}}+\mathcal{H}_{\text {tun }}$, where the Hamiltonian of the two Coulomb-coupled QDs is $\mathcal{H}_{\mathrm{DQD}}=\sum_{j} \varepsilon_{j} d_{j}^{\dagger} d_{j}+U d_{1}^{\dagger} d_{1} d_{2}^{\dagger} d_{2}$. Here, $\varepsilon_{j}$ is the position of the gate-controlled energy level in the $j$ th $\mathrm{QD}$, and $U$ is the interdot Coulomb interaction. The contacts are described by noninteracting reservoirs, $\mathcal{H}_{\text {leads }}=\sum_{\alpha j k} \xi_{\alpha j k} c_{\alpha j k}^{\dagger} c_{\alpha j k}$, where $c_{\alpha j k}^{\dagger}\left(c_{\alpha j k}\right)$ creates (annihilates) an electron in state $k$ of lead $\alpha=\{L, R\}$ and system $j$ with energy $\xi_{\alpha j k}$. In the drive system, the chemical potentials of the reservoirs are given by the applied bias voltage, $\mu_{\alpha} \equiv \mu_{\alpha 2}=\varepsilon_{F} \pm e V_{\alpha}$, while the reservoirs of the drag system are kept in equilibrium with $\mu_{\alpha 1}=$ $\varepsilon_{F}=0$. Finally, the dot-reservoir tunneling is represented by $\mathcal{H}_{\text {tun }}=\sum_{\alpha j k} \mathcal{V}_{\alpha j k} c_{\alpha j k}^{\dagger} d_{j}+$ H.c., where $\mathcal{V}_{\alpha j k}$ are the tunnel couplings.

We describe the QD system using NEGF where the contour-ordered dot Green's function (GF) $G_{j}\left(\tau, \tau^{\prime}\right)=$ $-i\left\langle T_{c} d_{j}(\tau) d_{j}^{\dagger}\left(\tau^{\prime}\right)\right\rangle$ is given by the usual Dyson equation with the irreducible self-energy, $\Sigma_{j}=\Sigma_{j \text {,tun }}+\Sigma_{j \text {,int }}$, having contributions from (i) the tunnel couplings to the leads, $\Sigma_{j, \text { tun }}\left(\tau, \tau^{\prime}\right)=\sum_{\alpha} \Sigma_{\alpha j, \operatorname{tun}}\left(\tau, \tau^{\prime}\right)=\sum_{\alpha k}\left|\mathcal{V}_{\alpha j k}\right|^{2} g_{\alpha j k}\left(\tau, \tau^{\prime}\right)$, where $g_{\alpha j k}$ is the unperturbed Green's function of the lead $\alpha j$, and (ii) the interdot Coulomb interaction, $\Sigma_{j, \text { int }}=\Sigma_{j, H}+\Sigma_{j, \mathrm{xc}}$. The latter is split into separate Hartree $(H)$ and exchange-correlation (xc) parts described in further detail below. Analytic continuation onto the real-time axis is performed with the Langreth rules [25].

The current in lead $\alpha j$ is defined as $I_{\alpha j}=-e \frac{d N_{\alpha j}}{d t}$, where $N_{\alpha j}$ is the total occupation of lead $\alpha j$, and can be expressed in terms of the dot GF and self-energies in Fourier space

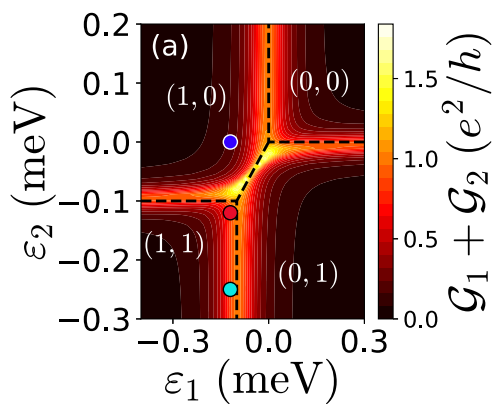

(b)

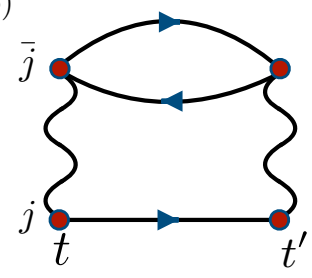

FIG. 2. (a) Stability diagram showing the energetically most favorable dot occupations $\left(n_{1}, n_{2}\right)$ vs level positions (gate voltage). The honeycomb vertex (dashed lines) connecting the two triple points at the center of the diagram is due to the interdot Coulomb interaction $U$. The color scale shows the sum of the linear conductances $\mathcal{G}_{1}+\mathcal{G}_{2}$ in the drive and drag systems. The dots mark the level configurations considered in Fig. 3. Parameters: $U=0.1 \mathrm{meV}, \gamma_{L 1}=\gamma_{R 1}=7 \mu \mathrm{eV}$, $\gamma_{L 2}=\gamma_{R 2}=25 \mu \mathrm{eV}, D_{\alpha j}=10 \mathrm{meV}$, lead coupling shifts $\tilde{\varepsilon}_{L 1}=$ $-\tilde{\varepsilon}_{R 1}=-2.5 \mathrm{meV}$, and $T=23 \mathrm{mK}$. (b) Feynman diagram for the single-bubble approximation to the xc part of interaction self-energy in Eq. (3).

as [25]

$$
\begin{aligned}
I_{\alpha j}= & \frac{e}{h} \int d \omega G_{j}^{r}(\omega) G_{j}^{a}(\omega) \\
& \times\left[\Sigma_{j}^{>}(\omega) \Sigma_{\alpha j, \text { tun }}^{<}(\omega)-\Sigma_{j}^{<}(\omega) \Sigma_{\alpha j, \text { tun }}^{>}(\omega)\right],
\end{aligned}
$$

where $\quad G_{j}^{r / a}(\omega)=\left[\omega-\varepsilon_{j}-\Sigma_{j}^{r / a}(\omega)\right]^{-1} \quad$ are the retarded/advanced dot Green's functions, and $\Sigma_{\alpha j, \operatorname{tun}}^{r / a}(\omega)=$ $\Lambda_{\alpha j}(\omega) \mp i \Gamma_{\alpha j}(\omega) / 2$ are the retarded/advanced tunneling self-energies, $\Lambda_{\alpha j}$ gives the shift of the levels due to the tunnel coupling, and $\Gamma_{\alpha j}$ is the lead hybridization function. The greater/lesser components are given by $\Sigma_{\alpha j, \text { tun }}^{<}(\omega)=$ $-i \Gamma_{\alpha j}(\omega) f_{\alpha j}(\omega) \quad$ and $\quad \Sigma_{\alpha j, \operatorname{tun}}^{>}(\omega)=i \Gamma_{\alpha j}(\omega)\left[1-f_{\alpha j}(\omega)\right]$, where $f_{\alpha j}(\omega)=1 /\left[1+\exp \left(\omega-\mu_{\alpha j}\right) / k_{B} T\right] \quad$ is the Fermi-Dirac distribution function.

From Eq. (2) we note that the interacting self-energy is, not surprisingly, instrumental for a nonzero drag current [31]. More specifically, it is the dynamic xc part of the interaction self-energy which is responsible for the drag since the Hartree part has $\Sigma_{j, H}^{>/<}=0$ [32]. On the other hand, the retarded/advanced Hartree self-energy, $\Sigma_{j, H}^{r, a}=U n_{\bar{j}}$, where $n_{j}=-i \int \frac{d \omega}{2 \pi} G_{j}^{<}(\omega)$ is the occupation of $\operatorname{dot} j, \bar{j} \neq j$, and $G_{j}^{<}=G_{j}^{r} \Sigma_{j}^{<\pi} G_{j}^{a}$, is essential for capturing the Coulomb coupling between the dots as it introduces an interaction-induced shift of the QD levels, $\varepsilon_{j} \rightarrow \varepsilon_{j}+U n_{\bar{j}}$.

The effect of the Coulomb coupling is evident from the stability diagram in Fig. 2(a), which shows the sum of the linear conductances $\mathcal{G}_{1}+\mathcal{G}_{2}, \mathcal{G}_{j}=d I_{j} / d V_{j}$, as a function of the level positions $\varepsilon_{j}$ for a DQD system in the regime $\Gamma \sim U$. As indicated in Fig. 2(a), the stability diagram maps out the energetically most favorable occupations of the QDs, and is characterized by the Coulomb-induced honeycomb vertex (dashed lines) connecting the triple points at $\varepsilon_{j}=-U$ and $\varepsilon_{j}=0$ [33].

For the xc part of the interaction self-energy, we adopt the single-bubble approximation illustrated by the Feynman 
diagram in Fig. 2(b), and given by

$$
\Sigma_{j, \mathrm{xc}}\left(\tau, \tau^{\prime}\right)=U^{2} G_{j}\left(\tau, \tau^{\prime}\right) P_{\bar{j}}\left(\tau, \tau^{\prime}\right), \quad \bar{j} \neq j,
$$

where $P_{j}\left(\tau, \tau^{\prime}\right)=G_{j}\left(\tau, \tau^{\prime}\right) G_{j}\left(\tau^{\prime}, \tau\right)$ is the nonequilibrium polarization bubble of the $j$ system. The greater and lesser components of the self-energy are $\Sigma_{j, \mathrm{xc}}^{>/<}\left(t, t^{\prime}\right)=U^{2} G_{j}^{>/<}\left(t, t^{\prime}\right) P_{\bar{j}}^{>/<}\left(t, t^{\prime}\right)$, where $P_{j}^{>/<}\left(t, t^{\prime}\right)=$ $G_{j}^{>/<}\left(t, t^{\prime}\right) G_{j}^{</>}\left(t^{\prime}, t\right)$. Generalization to more complicated self-energies, such as, e.g., the $G W$ approximation [32], is possible $[34,35]$. However, the $G W$ approximation is more relevant for extended systems where screening effects are important, and is therefore not expected to affect our findings below.

In the following, we pursue both analytic perturbative and numerical nonperturbative calculations [36]. In the numerical calculations, the xc self-energy in Eq. (3) is obtained from the Hartree GF. Since the latter itself depends on the dot occupations, the Hartree GF must be calculated self-consistently. We should stress that this procedure yields a conserving approximation with overall charge conservation respected [37], $\sum_{\alpha} I_{\alpha j}=0$, allowing us to define the drive and drag currents as $I_{j} \equiv\left(I_{L j}-I_{R j}\right) / 2$.

The drag current. We start by analyzing the drag arising from the self-energy in Eq. (3). Inserting in the general expression for the current in Eq. (2), the drag current can be written as [37]

$$
\begin{aligned}
I_{\text {drag }}= & \frac{e U^{2}}{h} \int d \omega \frac{d \omega^{\prime}}{2 \pi} \frac{\mathcal{A}_{1}(\omega) \mathcal{A}_{1}\left(\omega-\omega^{\prime}\right)}{\Gamma_{1}(\omega) \Gamma_{1}\left(\omega-\omega^{\prime}\right)} \\
& \times\left[\Gamma_{L 1}\left(\omega-\omega^{\prime}\right) \Gamma_{R 1}(\omega)-\Gamma_{L 1}(\omega) \Gamma_{R 1}\left(\omega-\omega^{\prime}\right)\right] \\
& \times f_{1}\left(\omega-\omega^{\prime}\right)\left[1-f_{1}(\omega)\right] P_{2}^{<}\left(\omega^{\prime}\right),
\end{aligned}
$$

where $\mathcal{A}_{j}(\omega)=-(1 / \pi) \operatorname{Im} G_{j}^{r}(\omega)$ is the dot spectral function and $\Gamma_{j}=\Gamma_{L j}+\Gamma_{R j}$.

Several important observations can be made from Eq. (4). First, in order for a nonzero drag current, the lead couplings in the drag QD must fulfill $\Gamma_{L 1}(\omega) \Gamma_{R 1}\left(\omega^{\prime}\right)-\Gamma_{L 1}\left(\omega^{\prime}\right)$ $\Gamma_{R 1}(\omega) \neq 0$, i.e., they cannot be proportional $\Gamma_{L 1}(\omega) \neq$ $C \Gamma_{R 1}(\omega)$. This result is consistent with previous works in the Coulomb blockade regime $[12,20]$. Second, the drag can be understood as arising from interaction-mediated creation and annihilation of electron-hole pair excitations in the drag and drive systems, and is driven by the finite-bias correlator $P_{2}^{<}$of the drive QD which can be identified as the quantum charge noise $P_{2}(\omega)$ in Eq. (1). At low temperature, i.e., $k_{B} T \ll e V$, where the drag QD predominantly absorbs energy from the drive QD due to Pauli blocking of emission processes, the quantum nature of the noise manifests itself in the fact that the drag is governed by the emission noise of the drive QD [23,38] given by $P_{2}(\omega>0)$ [37]. Lastly, the drag in Eq. (4) can be viewed as a rectification of the quantum charge noise in the drive system, which discerns it from the shot-noise-driven drag of Ref. [17] mentioned in the Introduction.

Weak-interaction regime. Having established the general properties of the drag, we next examine its behavior for weak interactions $(U \lesssim \Gamma)$.

In the small- $U$ limit, $U \ll \Gamma$, an explicit expansion of the drag current to second order in $U$ applies, and amounts to replacing $G_{j} \rightarrow G_{j}^{0}$, where $G_{j}^{0}$ is the noninteracting dot

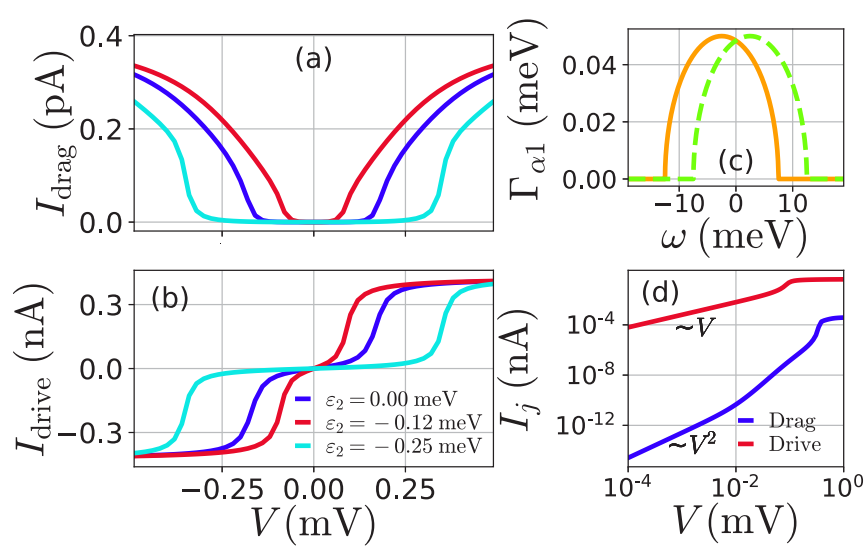

FIG. 3. (a) Drag current, and (b) drive current as a function of the applied bias voltage in the drive system for the level positions indicated with dots in Fig. 2(a). (c) Lead hybridization functions $\Gamma_{\alpha j}$ modeled by semielliptic bands. As illustrated, the bands in the left (solid) and right (dashed) leads of the drag system are shifted relative to each other in order to yield a nonzero drag. (d) Log-log plot of the drag and drive currents vs bias voltage showing the leading contributions of $V$. Same parameters as in Fig. 2 and $\varepsilon_{1}=$ $-0.12 \mathrm{meV}$.

GF, in Eq. (4). In this limit, our DQD system behaves similarly as two Coulomb-coupled single-channel QPCs with transmission coefficients $T_{j}=4 \Gamma_{j L} \Gamma_{j R} / \Gamma_{j}^{2}$, and the charge noise scales as $P_{2} \sim T_{2}$ for $k_{B} T \ll e V \ll \Gamma$ [37]. This should be contrasted with the qualitatively different $S_{2} \sim T_{2}\left(1-T_{2}\right)$ scaling of the shot noise [39], thus providing a means to distinguish between drag due to, respectively, direct Coulomb interactions and a circuit environment [17] by tuning the conductance $\mathcal{G}_{2}=\frac{e^{2}}{h} T_{2}$ of the drive system.

Next, we turn to numerical calculations of the drag using parameters mimicking the experiment in Ref. [12]. In order to fulfill the conditions for a nonzero drag, we model for convenience the lead couplings $\Gamma_{\alpha j}$ by semielliptic bands with bandwidth $D_{\alpha j}$ and a relative shift between the bands in the left and right contacts $\tilde{\varepsilon}_{L j}=-\tilde{\varepsilon}_{R j}$ of the drag system as illustrated in Fig. 3(c) [37].

Figures 3(a) and 3(b) show the calculated drag and drive currents for the level positions marked with the colored dots in Fig. 2(a). At low bias, the level of the drive dot is off resonance with respect to the chemical potentials and both the drive and drag currents are small. With increasing bias voltage, the level of the drive dot enters the conduction window and the onset of the drive current induces a current in the drag system. While the direction of the drive current follows the sign of the applied bias voltage, the direction of the drag is governed by the lead couplings. From Eq. (4), this can be traced back to the fact that $I_{\text {drag }}$ has no linear dependence on $V$ when the drive contacts have proportional lead couplings, $\Gamma_{L 2} \propto \Gamma_{R 2}$ [37]. As demonstrated in Fig. 3(d), the drag current at low bias thus increases as $I_{\text {drag }} \sim V^{2}$, emphasizing the inherent nonlinear nature of Coulomb drag in quantum dot systems [20]. The dependence of the currents on the QD levels at a fixed bias voltage of $V=0.5 \mathrm{mV}$ is shown in Fig. 4 . Here, the drive current in Fig. 4(a) shows a weak dependence on the drag level $\varepsilon_{1}$ due to the interaction-induced shift of 

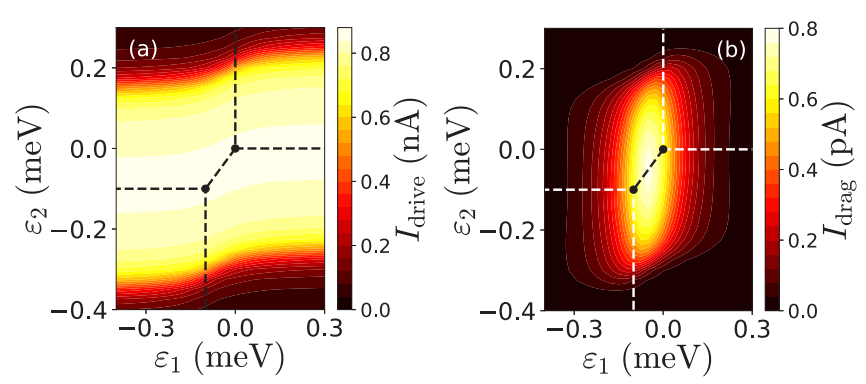

FIG. 4. (a) Drive current and (b) drag current as a function of the level positions $\varepsilon_{1}$ and $\varepsilon_{2}$ for an applied bias of $V=0.5 \mathrm{mV}$. All other parameters are the same as in Fig. 2.

the drive level upon changing the occupation of the drag QD. The drag current in Fig. 4(b) is only significant near the honeycomb vertex where the alignment between the QD levels and the chemical potentials allows for simultaneous interaction-induced electron-hole pair processes in the drive and drag system [cf. Fig. 1(b)]. Our results in Figs. 2(a) and 4 are in good qualitative agreement with the experimental measurements reported in Ref. [12] [their Figs. 2(b) and 2(c), and 2(e) and 2(f)].

Strong-interaction and Kondo regime. In the regime of strong interdot interaction, $U \gg \Gamma$, the DQD Hamiltonian can be mapped onto the Kondo Hamiltonian $\mathcal{H}_{\mathrm{K}}=$ $\mathcal{H}_{\text {dir }}+\mathcal{H}_{\text {ex }}$ by a Schrieffer-Wolff transformation [37,40] with the two QD states acting as a pseudospin $(\hat{S})$ exhibiting an orbital Kondo effect [6-8] at $T<T_{K}$, where $T_{K} \sim \sqrt{\Gamma U} \exp (-\pi U / 4 \Gamma)$ is the Kondo temperature [41]. Here, $\mathcal{H}_{\mathrm{dir}}=\sum_{\alpha \beta j k q} \mathcal{K}_{\alpha j, \beta j} c_{\alpha j k}^{\dagger} c_{\beta j q}$ is the potential scattering term responsible for elastic pseudospin-conserving cotunneling transitions, whereas the exchange interaction $\mathcal{H}_{\mathrm{ex}}=\sum_{\alpha \beta i j k q} \mathcal{J}_{\alpha i, \beta j} \hat{S}_{l} s_{i j}^{l} c_{\alpha i k}^{\dagger} c_{\beta j q}$ accounts for, e.g., inelastic pseudospin-flip cotunneling processes, and $\mathcal{K}_{\alpha i, \beta j}$ and $\mathcal{J}_{\alpha i, \beta j}$ are the effective couplings [27,37].

The importance of pseudospin (i.e., charge) fluctuations inherent to the orbital Kondo effect is evident already from the leading-order $I_{\text {drag }} \propto|\mathcal{J}|^{2}$ contribution to the drag. Performing a perturbative calculation of the drag at the particle-hole symmetric point $\left(\varepsilon_{1}=\varepsilon_{2}=-U / 2\right)$ [41], we find [37]

$$
\begin{aligned}
I_{\mathrm{drag}}= & \frac{e}{2 h} \sum_{\beta} \int d \omega \Delta \Gamma_{1} \Gamma_{\beta 2}\left|\frac{1}{\omega+U / 2}-\frac{1}{\omega-U / 2}\right|^{2} \\
& \times\left[f_{1}(\omega)-f_{\beta 2}(\omega)\right],
\end{aligned}
$$

where $\Delta \Gamma_{j}=\Gamma_{L j}-\Gamma_{R j}$. This can be viewed as drag due to nonlocal cotunneling processes where the pseudospin of the DQD is flipped in one coherent processes via virtual intermediate empty and filled states [20,21].

To get an indication of the corrections to the drag in Eq. (5) due to Kondo correlations, we show in Fig. 5 numerical results [based on Eq. (4)] for the drag at different temperatures at the particle-hole symmetric point. The spectral function $\mathcal{A}_{1}(\omega)$ of the drag dot in Fig. 5(a) clearly shows a low-temperature feature at the Fermi energy which resembles a Kondo peak [42-44]. Overall, the corresponding drag currents in Fig. 5(b) only show marginal changes relative to the perturbative result in Eq. (5) (dashed line). However, at low temperature and
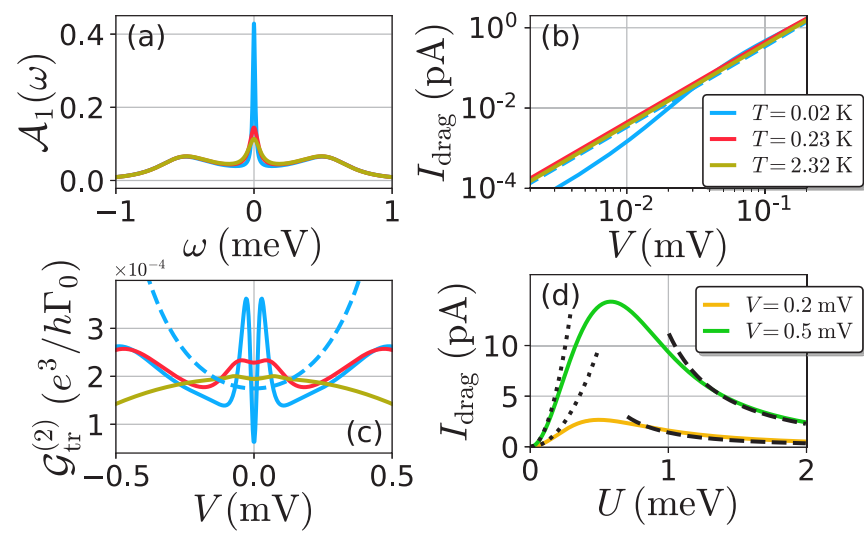

FIG. 5. (a) Spectral function of the drag system at zero drive bias, (b) drag current vs drive bias, and (c) second-order differential transconductance $\mathcal{G}_{\text {tr }}^{(2)}=d^{2} I_{\text {drag }} / d V^{2}$ of the drag current vs drive bias, all at the particle-hole symmetric point and for the temperatures indicated in (b). (d) Drag current as a function of the interdot interaction for two different bias voltages. Solid lines correspond to numerical calculations following Eq. (4), while dotted and dashed lines show, respectively, the leading-order contribution to Eq. (4) and the perturbative result in Eq. (5). Parameters: $U=1 \mathrm{meV}, \varepsilon_{1}=$ $\varepsilon_{2}=-U / 2, \gamma_{\alpha j}=0.05 \mathrm{meV}, \tilde{\varepsilon}_{L 1}=-\tilde{\varepsilon}_{R 1}=-4 \mathrm{meV}, \tilde{\varepsilon}_{L 2}=\tilde{\varepsilon}_{R 2}=$ 0 , and $D_{\alpha j}=10 \mathrm{meV}$.

low bias, Kondo correlations quench the charge fluctuations driving the drag which results in a suppression relative to the perturbative result. Due to the low-bias $I_{\mathrm{drag}} \sim V^{2}$ behavior of the drag, the effect of Kondo correlations is more noticeable in the second-order differential transconductance $\mathcal{G}_{\mathrm{tr}}^{(2)}=$ $d^{2} I_{\text {drag }} / d V^{2}$ shown in Fig. 5(c), which features a pronounced zero-bias anomaly in $\mathcal{G}_{\text {tr }}^{(2)}$ at low $T$. Finally, Fig. 5(d) shows the dependence of the drag on the strength of the interdot interaction at different bias voltages. For large values of $U$, the numerical results follow the $I_{\mathrm{drag}} \sim 1 / U^{2}$ behavior of the perturbative result of Eq. (5) (dashed lines), whereas the $I_{\text {drag }} \sim U^{2}$ behavior of the leading-order contribution to the drag in Eq. (3) is observed for small values of $U$ (dotted line). The two regimes are bridged by an intermediate region, $\Gamma \sim U$, with an optimal value of $U$ where $\left|I_{\text {drag }}\right|$ is maximized.

Conclusions. We have studied Coulomb drag across interaction regimes in Coulomb-coupled QD systems in the framework of the Keldysh NEGF technique. In agreement with previous works [12,19-21], we find that drag is an inherently nonlinear effect and that energy-dependent lead couplings are instrumental for the generation of a drag current. As we demonstrate, the drag is driven by the nonequilibrium charge fluctuations of the drive QD, and we discuss how the characteristics of the quantum noise allows one to differentiate the drag mechanism discussed here from drag induced by a circuit environment [17] experimentally. In the case of strong interdot interactions, the charge fluctuations are quenched by orbital Kondo correlations at low temperature and bias, which suppresses the drag current with respect to the lowest-order cotunneling-only drag [20]. In addition, we predict a clear signature of Kondo correlations in the second-order differential transconductance of the drag. Overall, our findings show that the Coulomb drag between quantum conductors is highly 
dependent on the interaction mediating the drag, and open the opportunity for further experimental studies of drag in QD systems.

Acknowledgments. We would like to thank J. Paaske for fruitful discussions. The work was supported by the CAIB predoctoral grant and the fellowships for research internships of the government of Balearic Islands. D.S. acknowl- edges support from Grant No. MAT2017-82639. K.K. acknowledges support from the European Union's Horizon 2020 research and innovation programme under the Marie Sklodowska-Curie Grant Agreement No. 713683 (COFUNDfellowsDTU). The Center for Nanostructured Graphene (CNG) is sponsored by the Danish National Research Foundation, Project DNRF103.
[1] G. Burkard, D. Loss, and D. P. DiVincenzo, Coupled quantum dots as quantum gates, Phys. Rev. B 59, 2070 (1999).

[2] T. C. Harman, P. J. Taylor, M. P. Walsh, and B. E. LaForge, Quantum dot superlattice thermoelectric materials and devices, Science 297, 2229 (2002).

[3] J. V. Koski, A. Kutvonen, I. M. Khaymovich, T. Ala-Nissila, and J. P. Pekola, On-Chip Maxwell's Demon as an InformationPowered Refrigerator, Phys. Rev. Lett. 115, 260602 (2015).

[4] H. Thierschmann, R. Sánchez, B. Sothmann, H. Buhmann, and L. W. Molenkamp, Thermoelectrics with Coulomb-coupled quantum dots, C. R. Phys. 17, 1109 (2016).

[5] H. Thierschmann, R. Sánchez, B. Sothmann, F. Arnold, C. Heyn, W. Hansen, H. Buhmann, and L. W. Molenkamp, Threeterminal energy harvester with coupled quantum dots, Nat. Nanotechnol. 10, 854 (2015).

[6] L. Borda, G. Zaránd, W. Hofstetter, B. I. Halperin, and J. von Delft, SU(4) Fermi Liquid State and Spin Filtering in a Double Quantum Dot System, Phys. Rev. Lett. 90, 026602 (2003).

[7] P. Jarillo-Herrero, J. Kong, H. S. J. van der Zant, C. Dekker, L. P. Kouwenhoven, and S. De Franceschi, Orbital Kondo effect in carbon nanotubes, Nature (London) 434, 484 (2005).

[8] S. Amasha, A. J. Keller, I. G. Rau, A. Carmi, J. A. Katine, H. Shtrikman, Y. Oreg, and D. Goldhaber-Gordon, PseudospinResolved Transport Spectroscopy of the Kondo Effect in a Double Quantum Dot, Phys. Rev. Lett. 110, 046604 (2013).

[9] A. Hamo, A. Benyamini, I. Shapir, I. Khivrich, J. Waissman, K. Kaasbjerg, Y. Oreg, F. von Oppen, and S. Ilani, Electron attraction mediated by Coulomb repulsion, Nature (London) 535, 395 (2016).

[10] B. N. Narozhny and A. Levchenko, Coulomb drag, Rev. Mod. Phys. 88, 025003 (2016).

[11] G. Shinkai, T. Hayashi, T. Ota, K. Muraki, and T. Fujisawa, Bidirectional current drag induced by two-electron cotunneling in coupled double quantum dots, Appl. Phys. Express 2, 081101 (2009).

[12] A. J. Keller, J. S. Lim, D. Sánchez, R. López, S. Amasha, J. A. Katine, H. Shtrikman, and D. Goldhaber-Gordon, Cotunneling Drag Effect in Coulomb-Coupled Quantum Dots, Phys. Rev. Lett. 117, 066602 (2016).

[13] D. Bischoff, M. Eich, O. Zilberberg, C. Rössler, T. Ihn, and K. Ensslin, Measurement back-action in stacked graphene quantum dots, Nano Lett. 15, 6003 (2015).

[14] C. Volk, S. Engels, C. Neumann, and C. Stampfer, Back action of graphene charge detectors on graphene and carbon nanotube quantum dots, Phys. Status Solidi B 252, 2461 (2015).

[15] N. A. Mortensen, K. Flensberg, and A.-P. Jauho, Coulomb Drag in Coherent Mesoscopic Systems, Phys. Rev. Lett. 86, 1841 (2001)
[16] N. A. Mortensen, K. Flensberg, and A.-P. Jauho, Mesoscopic fluctuations of Coulomb drag between quasiballistic onedimensional wires, Phys. Rev. B 65, 085317 (2002).

[17] A. Levchenko and A. Kamenev, Coulomb Drag in Quantum Circuits, Phys. Rev. Lett. 101, 216806 (2008).

[18] A. Borin, I. Safi, and E. Sukhorukov, The Coulomb drag effect induced by the third cumulant of current, Phys. Rev. B 99, 165404 (2019).

[19] R. Sánchez, R. López, D. Sánchez, and M. Büttiker, Mesoscopic Coulomb Drag, Broken Detailed Balance, and Fluctuation Relations, Phys. Rev. Lett. 104, 076801 (2010).

[20] K. Kaasbjerg and A.-P. Jauho, Correlated Coulomb Drag in Capacitively Coupled Quantum-Dot Structures, Phys. Rev. Lett. 116, 196801 (2016).

[21] J. S. Lim, D. Sánchez, and R. López, Engineering drag currents in Coulomb coupled quantum dots, New J. Phys. 20, 023038 (2018).

[22] R. Aguado and L. P. Kouwenhoven, Double Quantum Dots as Detectors of High-Frequency Quantum Noise in Mesoscopic Conductors, Phys. Rev. Lett. 84, 1986 (2000).

[23] Y. V. Nazarov and Y. M. Blanter, Quantum Transport: Introduction to Nanoscience (Cambridge University Press, Cambridge, UK, 2009).

[24] A. A. Clerk, M. H. Devoret, S. M. Girvin, F. Marquardt, and R. J. Schoelkopf, Introduction to quantum noise, measurement, and amplification, Rev. Mod. Phys. 82, 1155 (2010).

[25] H. Haug and A.-P. Jauho, Quantum Kinetics in Transport and Optics of Semiconductors (Springer, Berlin, 1998).

[26] E. A. Rothstein, O. Entin-Wohlman, and A. Aharony, Noise spectra of a biased quantum dot, Phys. Rev. B 79, 075307 (2009).

[27] A. C. Hewson, The Kondo Problem to Heavy Fermions (Cambridge University Press, Cambridge, UK, 1993).

[28] D. C. Ralph and R. A. Buhrman, Kondo-Assisted and Resonant Tunneling Via a Single Charge Trap: A Realization of the Anderson Model Out of Equilibrium, Phys. Rev. Lett. 72, 3401 (1994).

[29] W. G. van der Wiel, S. De Franceschi, T. Fujisawa, J. M. Elzerman, S. Tarucha, and L. P. Kouwenhoven, The Kondo effect in the unitary limit, Science 289, 2105 (2000).

[30] A. Rosch, J. Kroha, and P. Wölfle, Kondo Effect in Quantum Dots at High Voltage: Universality and Scaling, Phys. Rev. Lett. 87, 156802 (2001).

[31] The drag current (2) is zero for $\Sigma_{j \text {,int }}=0$, which follows straightforwardly from the identity $\Sigma_{L t \text {,tun }}^{>} \Sigma_{R t \text {, tun }}^{<}=$ $\Sigma_{L t \text {,tun }}^{<} \Sigma_{R t, \text { tun }}^{>}$for the tunnel self-energies in the unbiased drag system. 
[32] K. S. Thygesen and A. Rubio, Conserving $G W$ scheme for nonequilibrium quantum transport in molecular contacts, Phys. Rev. B 77, 115333 (2008).

[33] W. G. van der Wiel, S. De Franceschi, J. M. Elzerman, T. Fujisawa, S. Tarucha, and L. P. Kouwenhoven, Electron transport through double quantum dots, Rev. Mod. Phys. 75, 1 (2002).

[34] V. Moldoveanu and B. Tanatar, Coulomb drag in parallel quantum dots, Europhys. Lett. 86, 67004 (2009).

[35] C. Zhou and H. Guo, Coulomb drag between quantum wires: A nonequilibrium many-body approach, Phys. Rev. B 99, 035423 (2019).

[36] A. M. Lunde, A. De Marino, A. Schulz, R. Egger, and K. Flensberg, Electron-electron interaction effects in quantum point contacts, New J. Phys. 11, 023031 (2009).

[37] See Supplemental Material at http://link.aps.org/supplemental/ 10.1103/PhysRevB.100.081404 for details on the charge conservation, the final expression of the drag current, the charge noise expression in the wide-band limit, the model of the lead couplings, the Schrieffer-Wolff transformation and the perturbation expansion.

[38] U. Gavish, Y. Levinson, and Y. Imry, Detection of quantum noise, Phys. Rev. B 62, R10637(R) (2000).

[39] Y. M. Blanter and M. Büttiker, Shot noise in mesoscopic conductors, Phys. Rep. 336, 1 (2000).

[40] J. R. Schrieffer and P. A. Wolff, Relation Between the Anderson and Kondo Hamiltonians, Phys. Rev. 149, 491 (1966).

[41] A. Kaminski, Yu. V. Nazarov, and L. I. Glazman, Universality of the Kondo effect in a quantum dot out of equilibrium, Phys. Rev. B 62, 8154 (2000).

[42] B. Horvatić, D. Sokcević, and V. Zlatić, Finite-temperature spectral density for the Anderson model, Phys. Rev. B 36, 675 (1987).

[43] Q.-f. Sun and H. Guo, Double quantum dots: Kondo resonance induced by an interdot interaction, Phys. Rev. B 66, 155308 (2002).

[44] Z.-q. Bao, A.-M. Guo, and Q.-f. Sun, Orbital Kondo effect in a parallel double quantum dot, J. Phys.: Condens. Matter 26, 435301 (2014). 